\title{
Global epigenomic analysis indicates protocadherin-7 activates osteoclastogenesis by
}

\section{promoting cell-cell fusion}

Haruhiko Nakamura ${ }^{\mathrm{a}, \mathrm{b}}$, Tomoki Nakashima ${ }^{\mathrm{b}, \mathrm{c}}$, Mikihito Hayashi ${ }^{\mathrm{b}, \mathrm{d}}$, Naohiro Izawa ${ }^{\mathrm{a}}$, Tetsuro Yasui $^{\mathrm{a}}$, Hiroyuki Aburatani ${ }^{\mathrm{e}}$, Sakae Tanaka ${ }^{\mathrm{a}}$, and Hiroshi Takayanagi ${ }^{\mathrm{d}, \mathrm{f}^{*}}$

a Department of Orhopaedic Surgery, Faculty of Medicine, The University of Tokyo, Hongo 7-3-1, Bunkyo-ku, Tokyo 113-0033, Japan.

bepartment of Cell Signaling, Graduate school of Medical and Dental Science, Tokyo Medical and Dental University, Yushima 1-5-45, Bunkyo-ku, Tokyo 113-8549, Japan

'Japan Science and Technology Agency, PRESTO, Yushima 1-5-45, Bunkyo-ku, Tokyo 113-8549, Japan

dJapan Science and Technology Agency, ERATO, Takayanagi Osteonetwork Project, 7-3-1, Hongo, Bunkyo-ku, Tokyo, 113-0033, Japan

'Genome Science Division, Research Center for Advanced Science and Technology, The University of Tokyo, 4-6-1, Komaba, Meguro-ku, Tokyo, 153-8904, Japan

${ }^{\mathrm{f}}$ Department of Immunology, Graduate School of Medicine and Faculty of Medicine, The University of Tokyo, 7-3-1, Hongo, Bunkyo-ku, Tokyo, 113-0033, Japan 
* Corresponding author. Address: Department of Immunology, Graduate School of Medicine and Faculty of Medicine, The University of Tokyo, 7-3-1, Hongo, Bunkyo-ku, Tokyo, 113-0033, Japan. Fax: +81-3-5841-3450

E-mail address: takayana@m.u-tokyo.ac.jp (H. Takayanagi) 


\section{Abstract}

Gene expression is dependent not only on genomic sequences, but also epigenetic control, in which the regulation of chromatin by histone modification plays a crucial role. Histone H3 lysine 4 trimethylation (H3K4me3) and histone H3 lysine 27 trimethylation (H3K27me3) are related to transcriptionally activated and silenced sequences, respectively. Osteoclasts, the multinucleated cells that resorb bone, are generated by the fusion of precursor cells of monocyte/macrophage lineage. To elucidate the molecular and epigenetic regulation of osteoclast differentiation, we performed a chromatin immunoprecipitation sequencing (ChIP-seq) analysis for $\mathrm{H} 3 \mathrm{~K} 4 \mathrm{me} 3$ and $\mathrm{H} 3 \mathrm{~K} 27 \mathrm{me} 3$ in combination with RNA sequencing. We focused on the histone modification change from $\mathrm{H} 3 \mathrm{~K} 4 \mathrm{me} 3(+) \mathrm{H} 3 \mathrm{~K} 27 \mathrm{me} 3(+)$ to H3K4me3(+)H3K27me3(-) and identified the protocadherin-7 gene (Pcdh7) to be among the genes epigenetically regulated during osteoclastogenesis. Pcdh7 was induced by RANKL stimulation in an NFAT-dependent manner. The knockdown of Pcdh7 inhibited RANKL-induced osteoclast differentiation due to the impairment of cell-cell fusion, accompanied by a decreased expression of the fusion-related genes Dcstamp, Ocstamp and Atp6v0d2. This study demonstrates that Pcdh7 plays a key role in osteoclastogenesis by promoting cell-cell fusion. 


\section{Keywords}

Protocadherin-7 (Pcdh7)

Osteoclast

Epigenetics

RANKL

Cell-cell fusion 


\section{Introduction}

Bone homeostasis depends on the balance between the bone resorption by osteoclasts and formation by osteoblasts $[1,2]$. Osteoclasts are multinucleated giant cells that have bone resorption activity. They also play a critical role in various pathological conditions associated with bone loss such as osteoporosis, rheumatoid arthritis and cancer metastasis [1,2]. Therefore, a proper understanding of the regulatory mechanisms of osteoclastogenesis would provide a scientific basis for novel therapeutic strategies for such diseases.

Osteoclasts are differentiated from precursor cells of the monocyte/macrophage lineage in response to stimulation with macrophage colony-stimulating factor (M-CSF) and receptor activator of nuclear factor- $\kappa \mathrm{B}(\mathrm{NF}-\kappa \mathrm{B})$ ligand (RANKL) [1,2]. Nuclear factor of activated $\mathrm{T}$ cells (NFAT) c1, the master regulator of osteoclast differentiation, promotes mRNA expression of osteoclast related genes such as Ctsk (encoding Cathepsin K), Acp5 (tartrate-resistant acid phosphatase; TRAP), Dcstamp (dendritic cell specific transmembrane protein; DC-STAMP) and Ocstamp (osteoclast stimulatory transmembrane protein; OC-STAMP) in collaboration with other transcription factors, such as c-Fos and microphthalmia-associated transcription factor (MITF) [1,2]. Osteoclast precursors become multinucleated cells by cell-cell fusion [1-3]. TRAP-positive mononucleated cells, but no multinucleated osteoclasts, were observed in 
DC-STAMP or OC-STAMP-deficient mice [4,5]. Atp6v0d2-deficient mice exhibit an osteopetrotic phenotype due to impaired cell-cell fusion of osteoclast precursor cells [6]. These reports indicated that these molecules are important for cell-cell fusion during osteoclastogenesis, although the detailed molecular mechanisms at present remain unclear.

The level of gene expression is not only determined by the genomic sequence, but also by sequence-independent epigenetic regulation. Epigenetic regulatory mechanisms include histone modifications, such as methylation and acetylation, DNA methylation and microRNA-mediated regulation. Histone modifications are implicated in both gene activation and repression, depending on the specific lysine residue that is methylated or acetylated [7]. Histone H3 lysine 4 trimethylation ( $\mathrm{H} 3 \mathrm{~K} 4 \mathrm{me} 3)$ activates transcription by recruiting nucleosome remodeling enzymes and histone acetylases. In contrast, histone H3 lysine 27 trimethylation (H3K27me3) suppresses transcription by promoting chromatin compaction [8]. In osteoclastogenesis, it has been demonstrated that NFATc1 expression is regulated by a sequential change of histone modification from $\mathrm{H} 3 \mathrm{~K} 4 \mathrm{me} 3(+) \mathrm{H} 3 \mathrm{~K} 27 \mathrm{me} 3(+)$ to $\mathrm{H} 3 \mathrm{~K} 4 \mathrm{me} 3(+) \mathrm{H} 3 \mathrm{~K} 27 \mathrm{me} 3(-)$ [9], suggesting the importance of this histone modification change in the regulation of osteoclast genes.

Cadherin is a calcium-dependent adhesion protein that is classified into the classical cadherins, desmosomalcadherins and protocadherins [10]. In osteoclasts, it has been reported that E-cadherin expression was involved in the fusion of osteoclast precursor cells into mature 
multinucleated osteoclasts in vitro [11]. However, the contribution of other cadherins in osteoclastogenesis is as yet unclear.

In this study, we performed a chromatin immunoprecipiation sequencing (ChIP-seq) analysis of H3K4me3 and H3K27me3 in RANKL-stimulated osteoclast precursor cells and screened for genes characterized by a histone modification change from H3K4me3(+)H3K27me3(+) to H3K4me3(+)H3K27me3(-). We also analyzed mRNA expression by RNA sequencing and selected the genes highly expressed and upregulated during osteoclastogenesis. Then we focused on the role of Pcdh7 (encoding protocadherin-7) in osteoclastogenesis. Here we report that Pcdh7, a non-clustered protocadherin, which is involved in cell-cell adhesion and signal transduction in neural and cancer cells [10], plays an important role in osteoclastogenesis by regulating cell-cell fusion. 


\section{Materials and Methods}

In vitro osteoclastogenesis. In vitro osteoclastogenesis was performed as described previously with a minor modification [12-14]. Briefly, bone marrow cells were cultured with 10 ng/ml M-CSF (R\&D Systems, Minneapolis, MN) to obtain bone marrow-derived monocyte/macrophage precursor cells (BMMs). These cells or infected cells described in the knockdown analysis were cultured with 12.5 or 25 ng/ml RANKL (PeproTech, Rocky Hill, NJ) and M-CSF for 3 or 4 days. Cyclosporin A $(5 \mu \mathrm{g} / \mathrm{ml}$; Sigma-Aldrich, St. Louis, MO) was administered at the same time with RANKL. Osteoclastogenesis was evaluated by TRAP staining. TRAP-positive multinucleated cells (TRAP ${ }^{+}$MNCs; more than 3 nuclei) were counted unless otherwise described. These cells were incubated in the presence of HCS CellMask Deep Red stain (Life technologies, Carlsbad, CA) and Hoechst 33342 (Life technologies) to stain cell plasma membranes and nuclei. The numbers of cells and nuclei per each cell were counted using an imaging cytometer (IN Cell Analyzer 2000; GE Healthcare Bio-Sciences, Pittsburgh, PA) by detecting the fluorescence of stained plasma membranes and nuclei.

Chromatin immunoprecipitation (ChIP). ChIP was performed as described previously [9]. Cells were collected, pelleted and resuspended in sodium dodecyl sulfate (SDS) lysis buffer (50 $\mathrm{mM}$ Tris- $\mathrm{HCl}$ [pH 8.0], 1\% SDS, $10 \mathrm{mM}$ EDTA, and Complete Protease Inhibitor Cocktail 
[Roche Applied Science, Indianapolis, IN]). After sonication, centrifugation and removal of the control aliquot (whole-cell extract), supernatants were diluted 10-fold in ChIP dilution buffer (20 mM Tris- $\mathrm{HCl}[\mathrm{pH} 8.0], 150 \mathrm{mM} \mathrm{NaCl}, 2 \mathrm{mM}$ EDTA, and 1\% Triton X-100). Samples were incubated with protein A-Sepharose beads that had been preincubated with antibodies against H3K4me3 or H3K27me3. After washing, immunoprecipitates were incubated in elution buffer (50 mM Tris- $\mathrm{HCl}[\mathrm{pH} 8.0], 10 \mathrm{mM}$ EDTA and 1\% SDS) and treated with $1.5 \mathrm{mg}$ of pronase to obtain de-crosslinked DNA. Purified DNA was subjected to sequencing analysis.

ChIP sequencing. ChIP sequencing was performed as described previously [9]. Massively parallel sequencing of the ChIP products was performed using the Illumina cluster station and Genome Analyzer IIx (Illumina Inc., San Diego, CA). The sequences obtained were mapped to the reference mouse genome (build 36; National Center for Biotechnology Information). Peak detection was performed using Model-based analysis of ChIP-Seq [15]. RNA sequencing. Total RNA was extracted by ISOGEN (NIPPON GENE, Tokyo, Japan) following the manufacturer's protocol. Libraries were made by using TruSeq RNA Sample Preparation Kits (Illumina Inc.) according to the manufacturer's instructions. RNA sequencing was performed using the Illumina Genome Analyzer IIx.

Quantitative RT-PCR analysis. Total RNA was extracted as described in the previous paragraph. Real-time quantitative RT-PCR analysis was performed as described $[13,14,16]$. 
Briefly, First-strand cDNAs were synthesized from $0.5 \mu \mathrm{g}$ of total RNA using Superscript III reverse transcriptase (Life technologies). Quantitative RT-PCR analysis was performed with the LightCycler apparatus (Roche Applied Science) using SYBR Green Realtime PCR Master Mix (TOYOBO, Osaka, Japan). All primer sequences are available upon request. The level of mRNA expression was normalized with Gapdh expression.

Knockdown analysis. The knockdown of candidate genes was performed as described previously [13]. Briefly, short hairpin RNA (shRNA) duplexes were constructed based on the sequences obtained from the shRNA library of the RNAi Consortium. RNA targeting regions with a hairpin sequence were ligated into the RNAi-Ready pSIREN-RetroQ ZsGreen Vector (Clontech, Mountain View, CA) at the BamHI and EcoRI sites. The target sites were 5'-GATTCCGAGTGTCTCACTTAA-3' (shCd97-1), 5'-GCGTCTGTAACCTGGGATATA-3' $(\operatorname{shCd} 97-2)$, 5'-ACGTTCCCTCCATCGAAATTC-3' $(\operatorname{shPcdh} 7-1)$ 5'-CCAGTTGAGATTCACAGTAAT-3' (shPcdh7-2). pSIREN-RetroQ-ZsGreen-shCd97s, pSIREN-RetroQ-ZsGreen-shPcdh7s or a control pSIREN-RetroQ-ZsGreen-shRNA that specifically targets Luciferase was used to transfect BMMs. The retrovirus supernatants were obtained by transfecting the retroviral vectors into the Plat-E packaging cell line using Fugene HD Transfection Reagent (Promega, Madison, WI). BMMs were infected with retroviruses for 24 h before RANKL stimulation. We show the results of shCd97-1 and shPcdh7-1 as shPcdh7 
and shCd97. Moreover, the results of shCd97-2 and shPcdh7-2 had similar trends as shCd97-1

and shPcdh7-1, respectively.

Bone resorption assay. Bone resorption assays were performed as previously reported $[17,18]$. Briefly, infected mature osteoclasts were generated on collagen-coated dishes, as described in the knockdown analysis. These cells were harvested by treatment with Trypsin-EDTA (Life tecnologies). After cell counting, the same number of collected osteoclasts were seeded and cultured on dentin slices (Wako, Osaka, Japan). After 2 days of culture, the dentin slices were treated with $1 \mathrm{M} \mathrm{NH} 4 \mathrm{OH}$ along with sonication and stained with $0.5 \%$ toluidine blue. Resorption areas were quantified using image analysis (Microanalyzer; Japan Poladigital, Tokyo, Japan) and normalized to control.

Statistical analysis. Statistical analysis was performed using the two-tailed Student $t$ test unless otherwise described $\left({ }^{*} \mathrm{p}<0.05,{ }^{* *} \mathrm{p}<0.01,{ }^{* *} \mathrm{p}<0.001\right.$, NS, not significant, throughout the paper). All data are expressed as the mean \pm SEM ( $\mathrm{n}=3$ or more). Results are representative examples of more than 3 independent experiments. 


\section{Results}

Selection of candidate osteoclast regulatory genes by ChIP sequencing of H3K4me3 and H3K27me3.

To elucidate novel osteoclast regulatory genes that are epigenetically regulated during osteoclastogenesis, we performed ChIP sequencing of H3K4me3 and H3K27me3 in BMMs and mature osteoclasts generated by RANKL stimulation. Over $10 \%$ of the $\mathrm{H} 3 \mathrm{~K} 4 \mathrm{me} 3$ peaks were located at transcription start sites (TSS) and more than half of them were located less than $5 \mathrm{~kb}$ from TSS. In contrast, H3K27me3 peaks were rarely located at TSS and less than $20 \%$ of them were located in regions less than $5 \mathrm{~kb}$ from TSS (Fig. 1A).

We selected candidate genes which were marked by $\mathrm{H} 3 \mathrm{~K} 4 \mathrm{me} 3(+) \mathrm{H} 3 \mathrm{~K} 27 \mathrm{me} 3(+)$ in BMMs and H3K4me3(+)H3K27me3(-) in mature osteoclasts. The cut-off values of fold enrichment were 4 at $\mathrm{H} 3 \mathrm{~K} 4 \mathrm{me} 3$ (the mean in osteoclasts is 3.19) and 1 at $\mathrm{H} 3 \mathrm{~K} 27$ me3 (the mean in BMMs is 0.83) within $5 \mathrm{~kb}$ from TSS. H3K27me3(-) was defined as less than 0.8 (the mean in osteoclasts is 0.64 ).

In BMMs, 2006 genes were marked by $\mathrm{H} 3 \mathrm{~K} 4 \mathrm{me} 3(+) \mathrm{H} 3 \mathrm{~K} 27 \mathrm{me} 3(+)$. In mature osteoclasts, 7552 genes were marked by $\mathrm{H} 3 \mathrm{~K} 4 \mathrm{me} 3(+) \mathrm{H} 3 \mathrm{~K} 27 \mathrm{me} 3(-)$. It was determined that 579 genes had a histone modification change from H3K4me3(+)H3K27me3(+) to 
H3K4me3(+)H3K27me3(-) (Fig. 1B). These genes included Nfatc1, Nfkb2 (encoding the NF-kB p100 subunit), and Prdm1 (encoding the B lymphocyte-induced maturation protein 1; Blimp1), which are known to play crucial roles in osteoclast differentiation or function $[1,12]$, validating the selection method.

In the 579 candidate genes, we focused on the plasma membrane-bound proteins and, based on RNA sequencing results, selected genes which were expressed at a relative mRNA expression value over 20 (the mean of all gene expression is 16.2) in mature osteoclasts and were increased in mRNA expression by more than $20 \%$ after osteoclast differentiation. We identified Cav1, Cd82, Cd97, Pam, Pcdh7, Sdc1, Sema4d, Sigmar1, Slc11a2 and Tfrc as candidate genes (Fig. 1B). Cav1, Cd82, Pam, Sdc1, Sema4d and Tfrc were excluded from further study, since it is already reported that these genes are related to bone metabolism [19-24]. As shown in Fig. 2A, H3K4me3 and H3K27me3 enrichment was observed at the TSS in Nfatc1, Cd97 and Pcdh7, but not in Sigmar1 or Slc11a2, suggesting that only Cd97 and Pcdh7 are actually epigenetically regulated by this change in histone modification. These results prompted us to examine the role of Cd97 and Pcdh7 in osteoclastogenesis.

Regulation of Cd97 and Pcdh7 expression in osteoclastogenesis by NFAT. 
We generated osteoclasts in vitro by stimulating BMMs with RANKL and M-CSF and evaluated osteoclast formation by counting the number of $\mathrm{TRAP}^{+}$multinucleated cells. The expression of both Cd97 and Pcdh7 was increased during osteoclastogenesis (Fig. 2B). Cyclosporin A, an inhibitor of calcineurin, inhibited osteoclast differentiation and RANKL-induced Nfatc1 expression, as reported previously [25] (Fig. 2C, D). The expression of both Cd97 and Pcdh7 was also suppressed by cyclosporin A during osteoclastogenesis, suggesting that the expression of Cd97 and Pcdh7 is under the transcriptional control of NFAT (Fig. 2D).

Inhibition of osteoclastogenesis by the knockdown of Pcdh7.

To examine the function of CD97 and Pcdh7 in osteoclastogenesis, we performed a loss-of-function analysis by RNAi-mediated knockdown of CD97 and Pcdh7 using retroviral shRNA delivery system (Fig. 3A). We checked the expression of ZsGreen, a green fluorescent protein encoded by the retrovirus vector, using fluorescence microscopy (Fig. 3B). More than $80 \%$ of the cells were positive for ZsGreen, indicating that shRNA-expressing retroviral vectors were efficiently transduced into the osteoclast precursor cells. The mRNA expression of Cd97 and Pcdh7 in these cells after RANKL stimulation was significantly reduced by shCd97 and $\operatorname{shPcdh} 7$, respectively (Fig. 3C). Formation of $\mathrm{TRAP}^{+}$multinucleated cells was markedly 
inhibited by the knockdown of Pcdh7, but not CD97 (Fig. 3D). To investigate the role of CD97 and Pcdh7 in osteoclastic bone resorption, we performed a pit formation assay by culturing osteoclasts on dentine slices and knocked down CD97 or Pcdh7. Neither shCd97 nor shPcdh7 knockdown had any effect on the pit-forming activity of osteoclasts (Fig. 3E). These results suggest that Pcdh7 plays a specific role in the regulation of osteoclast differentiation.

Pcdh7 acts as a positive regulator of osteoclast differentiation by mediating the expression of cell-cell fusion related genes.

To gain insight into the mechanism underlying Pcdh7 regulation of osteoclastogenesis, we investigated the effect of Pcdh7 knockdown on the expression of genes implicated in osteoclast differentiation and function by quantitative RT-PCR (Fig. 4A). The expression of Mitf, Dcstamp, Ocstamp and Atp6v0d2 was significantly decreased by the Pcdh7 knockdown as compared to control (Fig. 4A). It is well documented that in osteoclast differentiation Dcstamp, Ocstamp and Atp6v0d2 encode cell-cell fusion related molecules and MITF regulates Dcstamp expression [26]. On the other hand, no significant difference was observed in the expression of Tnfrsf11a, Csf1r, Fos, Nfatc1, Prdm1, Irf8, Mafb, Ctsk, Clcn7 and Acp5 (Fig. 4A).

To clearly evaluate the cell fusion that takes place during osteoclastogenesis, we counted the number of osteoclasts containing a specific number of nuclei by a novel approach using an 
imaging cytometer. We obtained suitable power approximation curves demonstrating cell population possessing the nuclei (control; $y=27922 \mathrm{x}^{-2.84} ; \mathrm{R}^{2}=0.996, \operatorname{shPcdh} 7 ; \mathrm{y}=52532 \mathrm{x}^{-3.55}$; $\mathrm{R}^{2}=0.987$ ). Although there was no significant difference in the number of oligonucleated cells ( 8 or less nuclei), the multinucleated osteoclast (9 or more nuclei) number was significantly decreased by shPcdh7, suggesting that the knockdown of Pcdh7 inhibited the multinucleation of osteoclasts (Fig. 4B). These results demonstrate that Pcdh7 regulates cell-cell fusion during osteoclastogenesis by promoting the expression of cell-cell fusion related genes. 


\section{Discussion}

Histone modification regulates gene expression in the course of the differentiation of various cell types [7]. NFATc1, a critical transcription factor, is regulated by $\mathrm{H} 3 \mathrm{~K} 4 \mathrm{me} 3$ and H3K27me3 in osteoclastogenesis [9]. Using ChIP sequencing, we identified Pcdh7 to be under the control of a similar histone modification and it is one of the key osteoclast regulatory genes. Pcdh7, induced in an NFATc1-dependent manner, regulates osteoclast differentiation by promoting cell-cell fusion. A novel method was applied in this study to evaluate cell-cell fusion using an imaging cytometer.

Genes were screened based on the histone modification change from H3K4me3(+)H3K27me3(+) to H3K4me3(+)H3K27me3(-), because this change has reportedly been observed in the NFATc1 gene, the master gene of osteoclastogenesis. It is interesting to note that $\mathrm{H} 3 \mathrm{~K} 4$ is already trimethylated in osteoclast precursor cells and that demethylation of H3K27 functions as a switch for the initiation of gene induction. This type of regulation has been previously reported in the differentiation of ES cells to neuronal precursor cells [8]. Although it is reported that demethylation of $\mathrm{H} 3 \mathrm{~K} 27 \mathrm{me} 3$ in the promoter region of $N$ fatc 1 is regulated by Jmjd3 in osteoclastogenesis [9], it remains elusive which demethylase is involved in demethylation in Pcdh7. Osteoclastic genes such as Tnfrsf11a, Csf1r, Mitf, Ctsk, Clcn7 and Acp5 were marked by $\mathrm{H} 3 \mathrm{~K} 4 \mathrm{me} 3(+) \mathrm{H} 3 \mathrm{~K} 27 \mathrm{me} 3(-)$ in mature osteoclasts, but not by 
H3K4me3(+)H3K27me3(+) in osteoclast precursor cells (data not shown), suggesting that not all osteoclast genes are regulated by such histone modification change. In contrast, histone modification of anti-osteoclastogenic genes, such as Irf8 and Mafb, converted from H3K4me3(+)H3K27me3(+) to H3K4me3(-)H3K27me3(+) (data not shown). These results suggest that $\mathrm{H} 3 \mathrm{~K} 4$ and $\mathrm{H} 3 \mathrm{~K} 27$ modifications play an important role in temporal gene regulation during the course of osteoclastogenesis.

The process of cell-cell fusion generally consists of a number of distinct steps such as migration, recognition, adhesion and membrane fusion, which are regulated by various signaling pathways $[3,27]$. E-cadherin in osteoclasts and N-cadherin in myoblasts are implicated in the adhesion step, while M-cadherin in myoblasts mediates signal transduction in addition to the adhesion step during cell-cell fusion $[3,11,27]$. Previous reports on neuronal and cancer cells showed that non-clustered protocadherins, including Pcdh7, act as mediators of cell-cell adhesion and regulators of other molecules [10]. This study demonstrates a novel function of Pcdh7 in cell-cell fusion of osteoclasts and suggests that Pcdh7 is involved in the signal transduction or transcriptional regulation of cell-cell fusion genes.

Traditionally, osteoclast fusion has been analyzed by counting the multinucleated cell number with more than a certain threshold number of nuclei. The threshold is usually 2 to 4 , but there has been no consensus method for setting this threshold. Flow cytometric analysis has the 
capacity to determine the number of cells with a specific number of nuclei, but it is impossible to analyze large multinucleated osteoclasts by flow cytometry due to their excessive size. Here, we report the establishment of a novel method for evaluating osteoclastic cell fusion by means of an imaging cytometer. As shown in Fig. 4B, the exact number of cells containing a specific number of nuclei may be determined by this method. It is possible to obtain power approximation curves demonstrating cell populations possessing each specific nuclei count and to analyze the cell-cell fusion without setting the threshold beforehand. This method clearly showed that the fusion of oligonucleated osteoclasts (1-8 nuclei) was not influenced by shPcdh7, but the number of multinucleated osteoclasts ( 9 or more nuclei) was decreased by Pcdh7 knockdown.

Excessive bone resorption is the cause of pathologic bone loss in various osteopenic diseases. Denosumab, a human anti-RANKL neutralizing monoclonal antibody, inhibits osteoclastic bone resorption and is used for the treatment of both osteoporosis and bone metastasis in cancer [28]. Understanding the regulatory mechanisms of osteoclastogenesis and RANKL signal transduction will be helpful to the effort to develop new agents that inhibit osteoclastogenesis. This study reveals the importance of histone modification during osteoclast differentiation and the crucial role of Pcdh7 in osteoclast cell-cell fusion, providing a molecular basis for the development of novel therapeutic strategies. 


\section{Acknowledgements}

We thank T. Kitamura for providing Plat-E cell line. We also thank Y. Kadono, J. Hirose, S. Fukuse for reagents, discussion and technical assistance. This work was supported in part by grants for the ERATO Takayanagi Osteonetwork Project and PRESTO from Japan Science and

Technology Agency; Grant-in-Aids for Scientific Research (B), Young Scientist (A) and Challenging Exploratory Research from the Japan Society for the Promotion of Science (JSPS); and grants from Uehara Memorial Foundation, Terumo Life Science foundation, Takeda Medical Research Foundation, Mochida Memorial Foundation, Naito Foundation, Bristol-Myers RA Research Fund and Kanzawa Medical Research Foundation. 


\section{References}

[1] H. Takayanagi, Osteoimmunology: shared mechanisms and crosstalk between the immune and bone systems, Nat Rev Immunol 7 (2007) 292-304.

[2] T. Nakashima, M. Hayashi, H. Takayanagi, New insights into osteoclastogenic signaling mechanisms, Trends Endocrinol Metab 23 (2012) 582-590.

[3] M.J. Oursler, Recent advances in understanding the mechanisms of osteoclast precursor fusion, J Cell Biochem 110 (2010) 1058-1062.

[4] M. Yagi, T. Miyamoto, Y. Sawatani, K. Iwamoto, N. Hosogane, N. Fujita, K. Morita, K. Ninomiya, T. Suzuki, K. Miyamoto, Y. Oike, M. Takeya, Y. Toyama, T. Suda, DC-STAMP is essential for cell-cell fusion in osteoclasts and foreign body giant cells, $\mathrm{J}$ Exp Med 202 (2005) 345-351.

[5] H. Miyamoto, T. Suzuki, Y. Miyauchi, R. Iwasaki, T. Kobayashi, Y. Sato, K. Miyamoto, H. Hoshi, K. Hashimoto, S. Yoshida, W. Hao, T. Mori, H. Kanagawa, E. Katsuyama, A. Fujie, H. Morioka, M. Matsumoto, K. Chiba, M. Takeya, Y. Toyama, T. Miyamoto, Osteoclast stimulatory transmembrane protein and dendritic cell-specific transmembrane protein cooperatively modulate cell-cell fusion to form osteoclasts and foreign body giant cells, J Bone Miner Res 27 (2012) 1289-1297. 
[6] S.H. Lee, J. Rho, D. Jeong, J.Y. Sul, T. Kim, N. Kim, J.S. Kang, T. Miyamoto, T. Suda, S.K. Lee, R.J. Pignolo, B. Koczon-Jaremko, J. Lorenzo, Y. Choi, v-ATPase Vo subunit d2-deficient mice exhibit impaired osteoclast fusion and increased bone formation, Nat Med 12 (2006) 1403-1409.

[7] B.E. Bernstein, A. Meissner, E.S. Lander, The mammalian epigenome, Cell 128 (2007) 669-681.

[8] B.E. Bernstein, T.S. Mikkelsen, X. Xie, M. Kamal, D.J. Huebert, J. Cuff, B. Fry, A. Meissner, M. Wernig, K. Plath, R. Jaenisch, A. Wagschal, R. Feil, S.L. Schreiber, E.S. Lander, A bivalent chromatin structure marks key developmental genes in embryonic stem cells, Cell 125 (2006) 315-326.

[9] T. Yasui, J. Hirose, S. Tsutsumi, K. Nakamura, H. Aburatani, S. Tanaka, Epigenetic regulation of osteoclast differentiation: possible involvement of Jmjd3 in the histone demethylation of Nfatc1, J Bone Miner Res 26 (2011) 2665-2671.

[10] S.Y. Kim, S. Yasuda, H. Tanaka, K. Yamagata, H. Kim, Non-clustered protocadherin, Cell Adh Migr 5 (2011) 97-105.

[11] G. Mbalaviele, H. Chen, B.F. Boyce, G.R. Mundy, T. Yoneda, The role of cadherin in the generation of multinucleated osteoclasts from mononuclear precursors in murine marrow, J Clin Invest 95 (1995) 2757-2765. 
[12] K. Nishikawa, T. Nakashima, M. Hayashi, T. Fukunaga, S. Kato, T. Kodama, S. Takahashi, K. Calame, H. Takayanagi, Blimp1-mediated repression of negative regulators is required for osteoclast differentiation, Proc Natl Acad Sci U S A 107 (2010) 3117-3122.

[13] M. Hayashi, T. Nakashima, T. Kodama, A.P. Makrigiannis, N. Toyama-Sorimachi, H. Takayanagi, Ly49Q, an ITIM-bearing NK receptor, positively regulates osteoclast differentiation, Biochem Biophys Res Commun 393 (2010) 432-438.

[14] M. Hayashi, T. Nakashima, M. Taniguchi, T. Kodama, A. Kumanogoh, H. Takayanagi, Osteoprotection by semaphorin 3A, Nature 485 (2012) 69-74.

[15] Y. Zhang, T. Liu, C.A. Meyer, J. Eeckhoute, D.S. Johnson, B.E. Bernstein, C. Nusbaum, R.M. Myers, M. Brown, W. Li, X.S. Liu, Model-based analysis of ChIP-Seq (MACS), Genome Biol 9 (2008) R137.

[16] T. Nakashima, M. Hayashi, T. Fukunaga, K. Kurata, M. Oh-Hora, J.Q. Feng, L.F. Bonewald, T. Kodama, A. Wutz, E.F. Wagner, J.M. Penninger, H. Takayanagi, Evidence for osteocyte regulation of bone homeostasis through RANKL expression, Nat Med 17 (2011) 1231-1234.

[17] K. Sato, A. Suematsu, T. Nakashima, S. Takemoto-Kimura, K. Aoki, Y. Morishita, H. Asahara, K. Ohya, A. Yamaguchi, T. Takai, T. Kodama, T.A. Chatila, H. Bito, H. 
Takayanagi, Regulation of osteoclast differentiation and function by the CaMK-CREB pathway, Nat Med 12 (2006) 1410-1416.

[18] E. Idrus, T. Nakashima, L. Wang, M. Hayashi, K. Okamoto, T. Kodama, N. Tanaka, T. Taniguchi, H. Takayanagi, The role of the BH3-only protein Noxa in bone homeostasis, Biochem Biophys Res Commun 410 (2011) 620-625.

[19] N. Hada, M. Okayasu, J. Ito, M. Nakayachi, C. Hayashida, T. Kaneda, N. Uchida, T. Muramatsu, C. Koike, M. Masuhara, T. Sato, Y. Hakeda, Receptor activator of NF-kB ligand-dependent expression of caveolin-1 in osteoclast precursors, and high dependency of osteoclastogenesis on exogenous lipoprotein, Bone 50 (2012) 226-236.

[20] K. Iwai, M. Ishii, S. Ohshima, K. Miyatake, Y. Saeki, Expression and function of transmembrane-4 superfamily (tetraspanin) proteins in osteoclasts: reciprocal roles of Tspan-5 and NET-6 during osteoclastogenesis, Allergol Int 56 (2007) 457-463.

[21] R.S. Birnbaum, G.A. Howard, B.A. Roos, Ontogeny of peptidylglycine alpha-amidating monooxygenase activity in rapidly mineralizing bone from neonatal mouse, Endocrinology 124 (1989) 3134-3136.

[22] T. Kelly, L.J. Suva, K.M. Nicks, V. MacLeod, R.D. Sanderson, Tumor-derived syndecan-1 mediates distal cross-talk with bone that enhances osteoclastogenesis, J Bone Miner Res 25 (2010) 1295-1304. 
[23] T. Negishi-Koga, M. Shinohara, N. Komatsu, H. Bito, T. Kodama, R.H. Friedel, H.

Takayanagi, Suppression of bone formation by osteoclastic expression of semaphorin 4D, Nat Med 17 (2011) 1473-1480.

[24] K.A. Ishii, T. Fumoto, K. Iwai, S. Takeshita, M. Ito, N. Shimohata, H. Aburatani, S. Taketani, C.J. Lelliott, A. Vidal-Puig, K. Ikeda, Coordination of PGC-1 $\beta$ and iron uptake in mitochondrial biogenesis and osteoclast activation, Nat Med 15 (2009) 259-266.

[25] H. Takayanagi, S. Kim, T. Koga, H. Nishina, M. Isshiki, H. Yoshida, A. Saiura, M. Isobe, T. Yokochi, J. Inoue, E.F. Wagner, T.W. Mak, T. Kodama, T. Taniguchi, Induction and activation of the transcription factor NFATc1 (NFAT2) integrate RANKL signaling in terminal differentiation of osteoclasts, Dev Cell 3 (2002) 889-901.

[26] N. Courtial, J.J. Smink, O.N. Kuvardina, A. Leutz, J.R. Gothert, J. Lausen, Tal1 regulates osteoclast differentiation through suppression of the master regulator of cell fusion DC-STAMP, FASEB J 26 (2012) 523-532.

[27] S.M. Abmayr, G.K. Pavlath, Myoblast fusion: lessons from flies and mice, Development 139 (2012) 641-656.

[28] S.R. Cummings, J. San Martin, M.R. McClung, E.S. Siris, R. Eastell, I.R. Reid, P. Delmas, H.B. Zoog, M. Austin, A. Wang, S. Kutilek, S. Adami, J. Zanchetta, C. Libanati, S. 
Siddhanti, C. Christiansen, Denosumab for prevention of fractures in postmenopausal women with osteoporosis, N Engl J Med 361 (2009) 756-765. 


\section{Figure Legends}

Fig. 1. Profiles of $\mathrm{H} 3 \mathrm{~K} 4 \mathrm{me} 3$ and $\mathrm{H} 3 \mathrm{~K} 27 \mathrm{me} 3$ modification changes during osteoclastogenesis obtained by ChIP sequencing. (A) Genomic distribution of the histone modification sites. The peak locations of $\mathrm{H} 3 \mathrm{~K} 4 \mathrm{me} 3$ and $\mathrm{H} 3 \mathrm{~K} 27 \mathrm{me} 3$ relative to gene annotations are shown. TSS; transcription start sites. (B) Flowchart of the identification of candidate genes by ChIP sequencing for $\mathrm{H} 3 \mathrm{~K} 4 \mathrm{me} 3$ and $\mathrm{H} 3 \mathrm{~K} 27 \mathrm{me} 3$ in combination with RNA sequencing.

Fig. 2. Selection of the candidate genes which are epigenetically regulated during osteoclastogenesis. (A) ChIP sequencing for H3K4me3 and H3K27me3 around the transcription start sites of the candidate genes and Nfatc1. (B) The mRNA expression of Nfatc1, Cd97 and Pcdh7 during osteoclastogenesis. All statistical analysis was performed compared to day 0. (C) Effect of cyclosporine A (CsA) on osteoclastogenesis. (D) Effect of cyclosporine A (CsA) on the mRNA expression of Nfatc1, Cd97 and Pcdh7 during osteoclastogenesis. $(* \mathrm{p}<0.05, * * \mathrm{p}<0.01$, control vs CsA on day 3)

Fig. 3. Effect of CD97 and Pcdh7 knockdown on osteoclast differentiation and bone resorption.

(A) A schematic of the in vitro osteoclast culture system and retroviral infection. (B) Phase 
contrast microscopy (upper panel) and fluorescence microscopy (lower panel) of bone marrow cells cultured with RANKL for 3 days after retrovirus infection. Retrovirus-infected cells expressed ZsGreen. (C) Relative mRNA expression of Cd97 and Pcdh7 in osteoclasts after shRNA-mediated knockdown. (D) Effect of shRNA for Cd97 and Pcdh7 on osteoclast differentiation. (E) Effect of the retrovirus-mediated introduction of the shRNA for Cd97 and Pcdh7 on the pit-forming activity of osteoclasts.

Fig. 4. Role of Pcdh7 in RANKL-induced osteoclastogenesis. (A) Effect of Pcdh7 knockdown on the expression of osteoclastic genes. $(* p<0.05$, control vs shPcdh7 on day 3 ) (B) Effect of pcdh7 knockdown on osteoclast fusion analyzed by an imaging cytometer. The X-axis represents the numbers of nuclei per cell. The Y-axis represents the cell populations with a specific number of nuclei in each cell. Curves were fit with the cell numbers per intracellular nucleus number based on power approximation. Statistical analysis was performed compared control versus $\underline{\text { shPcdh7 using Two-way ANOVA after logarithmic transformation followed by Bonferroni test }}$ $\underline{(\mathrm{n}=4)}$ 
Figure 1

A

H3K4me3

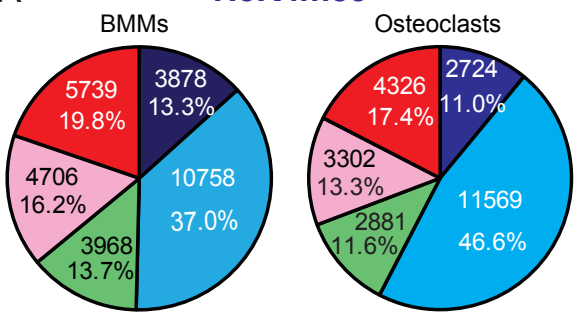

B

BMMs

Osteoclasts

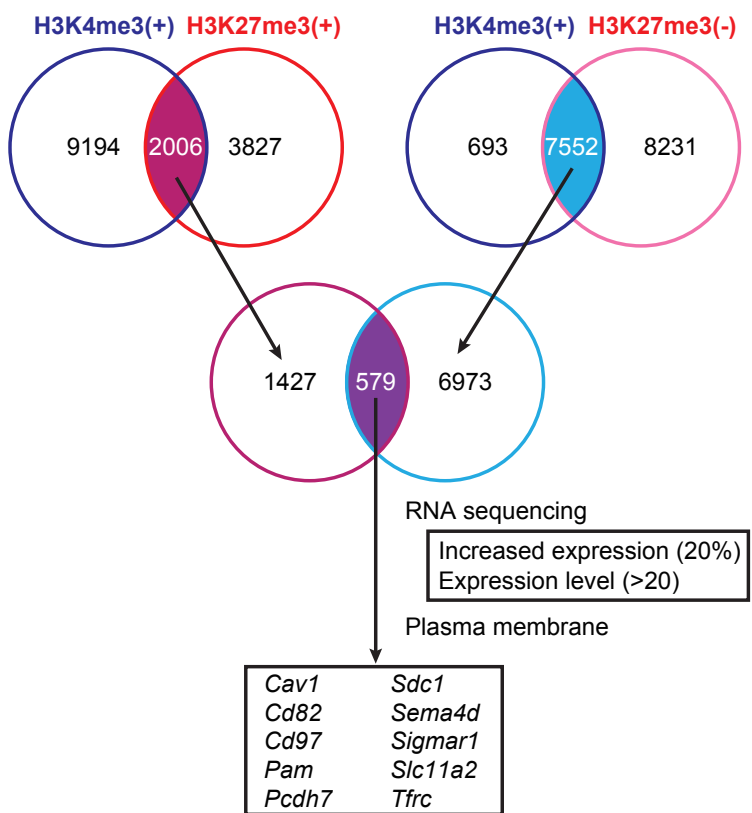

$0-5 \mathrm{~kb}$ from TSS

$\square 5$ - 15kb from TSS

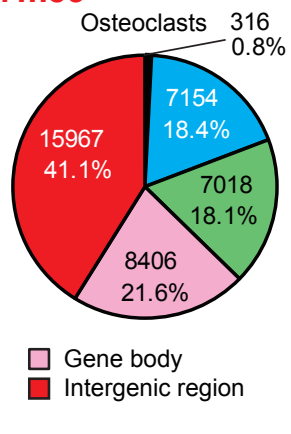

Pcdh7 Tfrc 
Figure 2
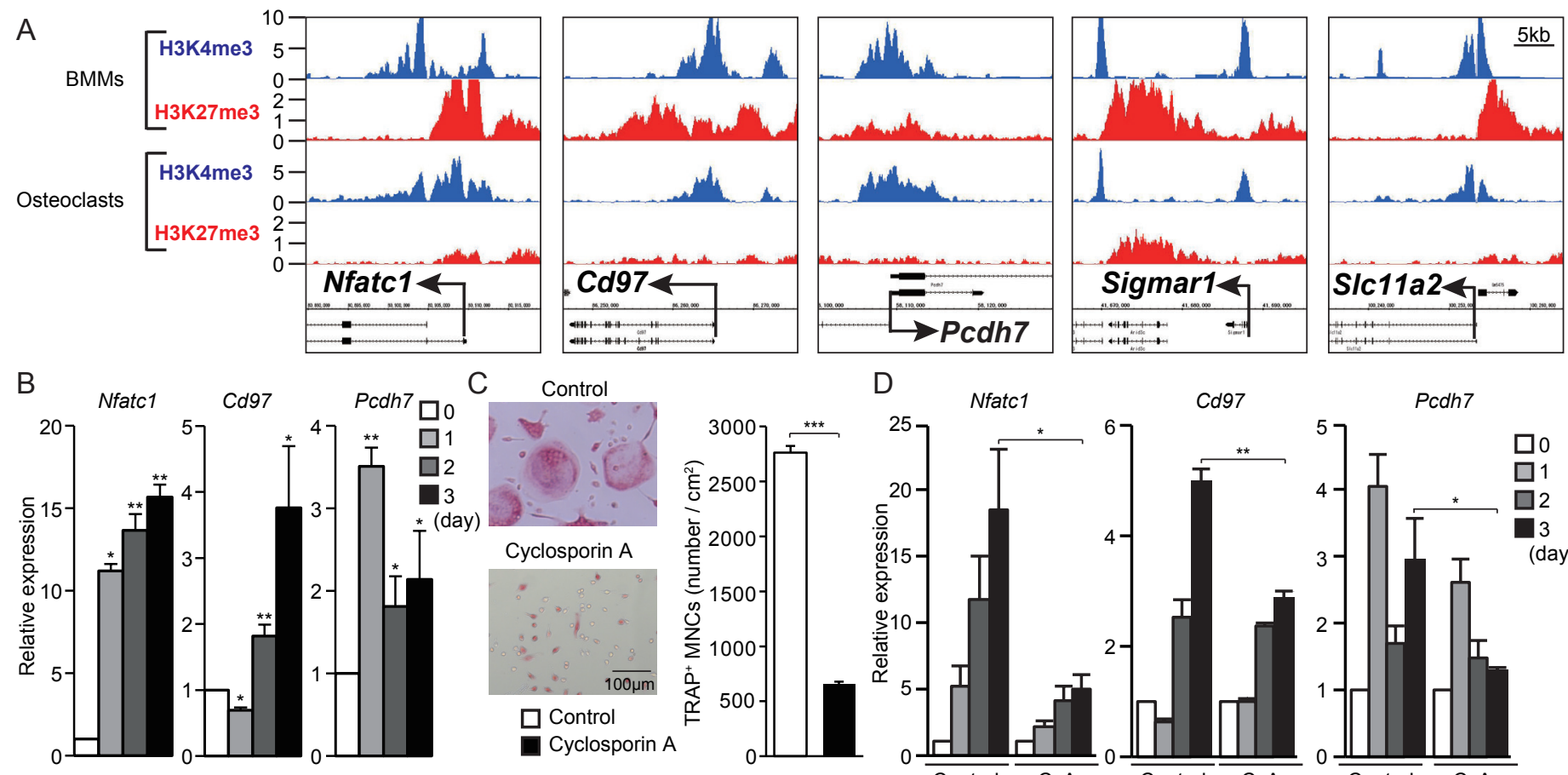

Cd97

Pcdh7
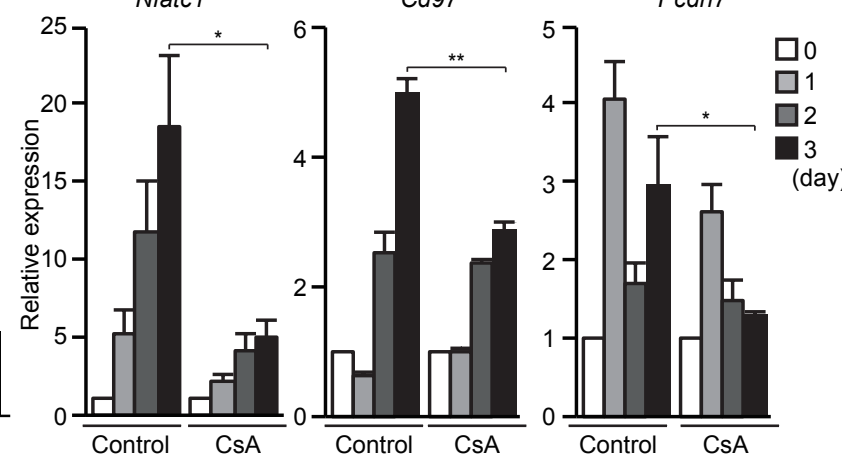
Figure 3

A

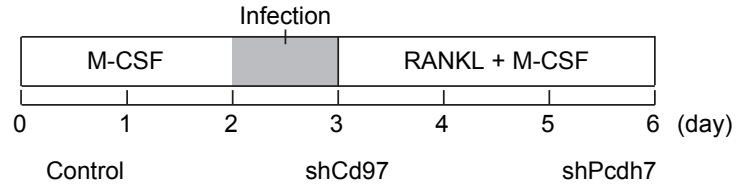

B
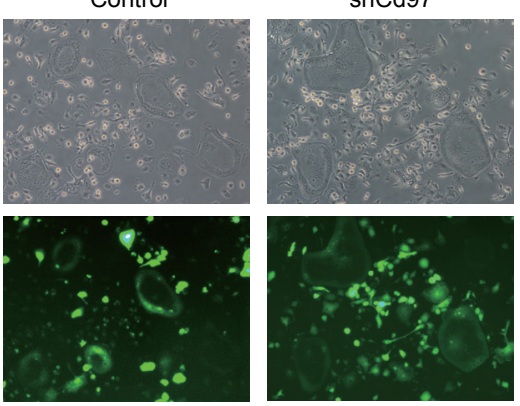

shCd97

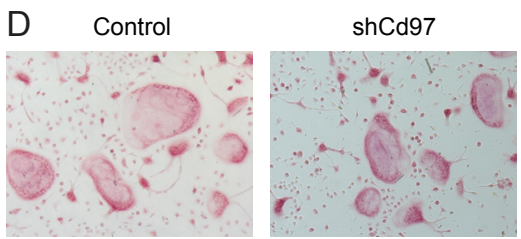

$\operatorname{shCd97}$

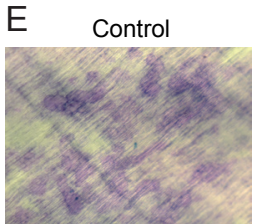

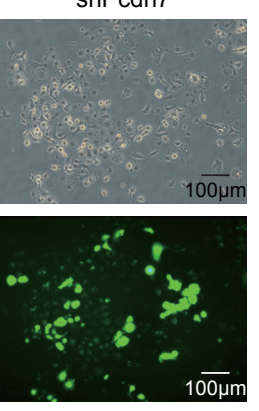

$\operatorname{shPcdh} 7$

$\operatorname{shPcdh} 7$

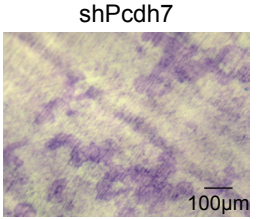

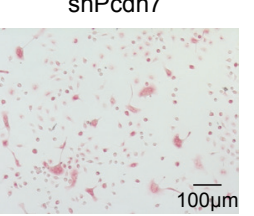

C

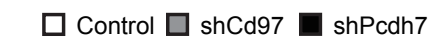

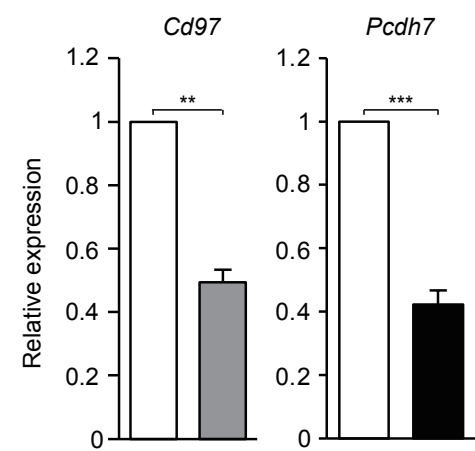

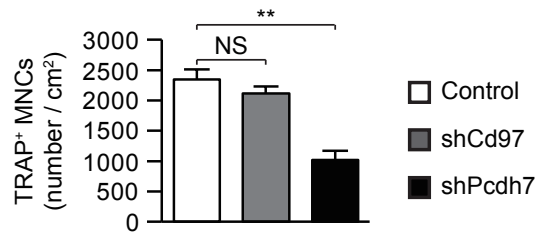

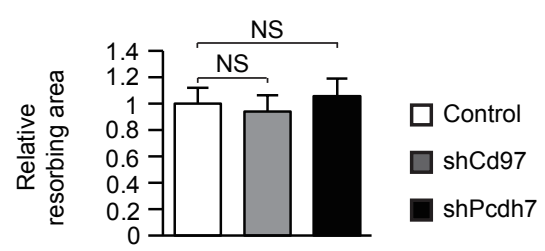


Figure 4

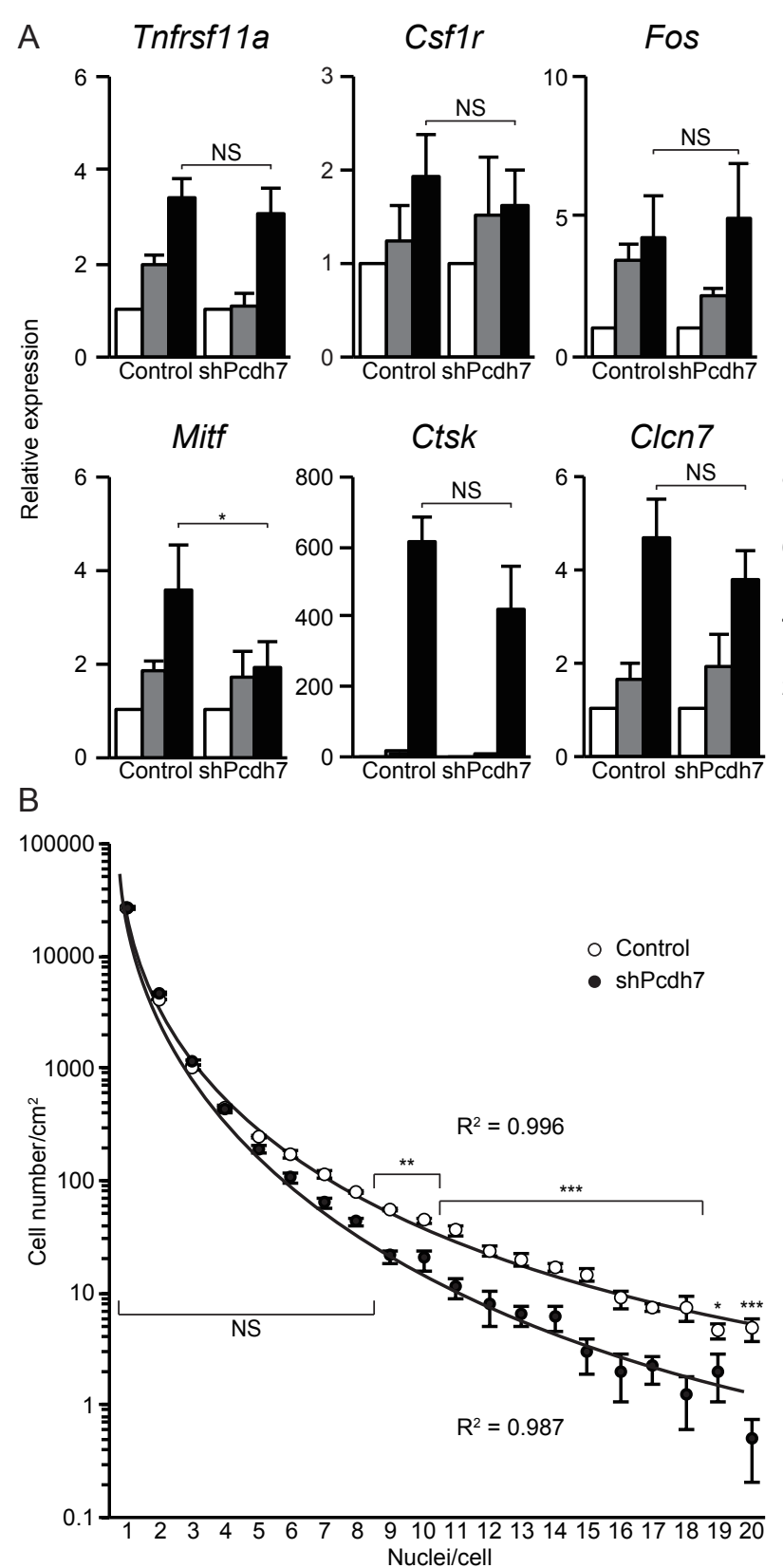

